\title{
Retrospective Analysis of Septic Arthritis Caused by Intra- Articular Viscosupplementation and Steroid Injections in a Single Outpatient Center
}

\author{
Mujtaba Mohamed ${ }^{a}$, Swapnil Patel ${ }^{\mathrm{a}}$, Kathy Plavnik ${ }^{\mathrm{a}}$, Edward Liu ${ }^{\mathrm{a}, \mathrm{b}}$, \\ Kathleen Casey ${ }^{\mathrm{a}}$, Mohammad A. Hossain ${ }^{\mathrm{a}}$
}

\begin{abstract}
Background: Septic arthritis is defined by the presence of pathogen(s) in a joint by direct inoculation or hematogenous spread. Most common organisms include Staphylococcus aureus and Escherichia coli. Clinical presentation is fever, warmth and night pain, with most common joints involved being the knee and hip. Iatrogenic septic arthritis is an uncommon complication of intra-articular injection for osteoarthritis yet its complications can be devastating. We aim to highlight ten cases of iatrogenic septic arthritis in retrospective study reviewing symptoms, signs, laboratory data, causing organisms and reasons leading to those infections.
\end{abstract}

Methods: Retrospective analysis of charts of ten patients who were admitted to Jersey Shore University Medical Center with diagnosis of iatrogenic septic arthritis.

Results: Average age of patients is 69.9 years. Most common comorbidities seen in our patient were hypertension and diabetes mellitus. The most common intra-articular agents that were injected were cortisone and Synvisc. The mean incubation period was 11.9 days. Most common presenting symptoms were joint pain and swelling. The most common organism isolated in cultures was Streptococcus mitis. A total of $100 \%$ of patients underwent surgical intervention for septic arthritis. One case was complicated by sepsis.

Conclusions: Iatrogenic septic arthritis is not common; however its complications can be catastrophic to patients. Improper sterile techniques and untrained physicians are the main risks factors for this complication. Physicians should take proper sterile measures to avoid complications of intra-articular injections.

Keywords: Septic arthritis; Intra-articular injections; Steroid injections

Manuscript submitted April 13, 2019, accepted May 2, 2019

aDepartment of Medicine, Jersey Shore University Medical Center, Hackensack Meridian Health, Neptune, NJ 07753, USA

${ }^{b}$ Corresponding Author: Edward Liu, Department of Medicine, Jersey Shore University Medical Center, Hackensack Meridian Health, Neptune, NJ 07753 , USA.Email: Edward.liu@hackensackmeridian.org

doi: https://doi.org/10.14740/jocmr3838

\section{Introduction}

Septic arthritis is defined by the presence of pathogen(s) in a joint by direct inoculation or hematogenous spread. It is a relatively uncommon disease with an annual incidence of 2 10 cases per 100,000 [1-2]. Delayed diagnosis or suboptimal treatment is associated with irreversible joint damage and permanent disability. It has about $10 \%$ mortality and significant morbidity [3]. Staphylococcus aureus including methicillinresistant Staphylococcus aureus (MRSA) is the most common pathogen (51.4\%) followed by gram negative enteric bacillus such as E.coli (5.7\%), which is more prevalent in elderly patients, immunocompromised patients, and patients with intravascular devices, urinary catheters and Streptococcus pyogenes (4.7\%) [1-2]. Risk factors for septic arthritis are immunosuppression $(30 \%)$, previous knee surgery (14\%), intravenous drug use, joint prostheses, alcoholism, diabetes, previous steroid injections, and cutaneous ulcers [2, 4-7]. Common clinical presentations are fever $(30 \%)$, local warmth $(100 \%)$, night pain $(85 \%)$, and common laboratory abnormalities are elevated erythrocyte sedimentation rate (ESR) $(85 \%)$ and C-reactive protein (CRP) (100\%) [8]. A total of $22 \%$ of septic arthritis are poly-articular which often reflect bacteremia seeding multiple joints. The most common joints involved are the knee (48\%) and hip (21\%) [7]. Key for diagnosis of septic arthritis is microscopic analysis and cultures of the synovial fluid [5]. Gram stain and microscopy of synovial fluid has positive results in $50 \%$ [5]. Synovial fluid analysis of white blood cells (WBCs) $>50,000$ has $50 \%$ sensitivity to diagnose septic arthritis.

Intra-articular injections have become popular as a treatment option for painful osteoarthritis where patients cannot tolerate non-steroidal anti-inflammatory drugs (NSAIDs) or inadequate pain control by NSAIDs or other analgesics [8]. Results from meta-analysis showed that intra-articular steroids and hyaluronic acid are associated with improvement of pain and functionality although it is not risk-free [9-10]. Risk factors associated with increasing chance of knee infection are corticosteroid injections, history of rheumatoid arthritis or other joint abnormalities, and injections performed by general practitioners [8]. In March 2017, an outbreak of multiple cases of septic arthritis occurred in NJ, USA. A total of 41 cases were identified by New Jersey Department of Health (NJDOH) [11] and the outbreak was mainly attributed to intra-articular injec- 
tions administered at a single outpatient practice in NJ. Ten of these cases were treated in our hospital [11]. We retrospectively analyzed these cluster cases that were managed in our hospital to identify the major factors associated with this outbreak and to prevent future incidence.

\section{Materials and Methods}

Study was conducted at Jersey Shore University Medical Center (JSUMC), Neptune, NJ, USA. The medical records of patients with acute septic arthritis who had knee injection at local osteoarthritis clinic and was subsequently seen by Meridian Infectious Diseases as inpatient at JSUMC or outpatient from October 2016 to May 2017 were reviewed. Data was analyzed retrospectively from medical records at JSUMC where these patients were seen.

Demographic characteristics (age, gender), past medical history, clinical variables on admission, time of onset of symptoms, incubation period, bacterial pathogen, length of stay at the hospital, treatment duration, need for surgery, need for peripherally inserted central catheter (PICC) line, complications of hospitalization, and outcome (complete resolution, discharge to home, and rehabilitation) were reviewed.

Meridian Institutional review board approval was obtained for this study. All study procedures were carried out in accordance with the Declaration of Helsinki regarding research involving human subjects. All data analysis was conducted with Microsoft excel software.

\section{Results}

Demographics of patients who presented at our medical center are shown in Table 1. Most of the patients were ages between 61 and 70 with a mean age of 69.9 years. The most common comorbidities seen in our patient were hypertension (four patients) and diabetes mellitus (two patients). All these cases had more than one intra-articular injection at the outpatient facility. The most common intra-articular agents that were injected were cortisone and Synvisc.

The mean incubation period for these patients was 11.9 days. All patients in our case series present with joint pain and swelling, with other reported symptoms including fever (two patients) and decrease range of motion (three patients). Mean ESR and CRP levels were $52.6 \mathrm{~mm} / \mathrm{h}$ and $10.3 \mathrm{mg} / \mathrm{dL}$ respectively. Two patients had positive calcium pyrophosphate deposition (CPPD) crystals and one had positive urate crystals as it appears in Table 2. The most common organism isolated in cultures was Streptococcus mitis (three patients) shown in Table 2, with other organisms all being oral flora. Five patients did not have an organism identified on cultures. Of these five patients, one patient started on empirical antibiotics (ampicillin-sulbactam) prior to joint tap, and one patient had negative cultures on the first admission but with a positive culture on the second admission. In one patient, antibiotics were stopped due to low suspicion of septic arthritis.

The average length of stay for these ten patients was 8
Table 1. Demographic and Clinical Characteristics of the Patients $(n=10)$

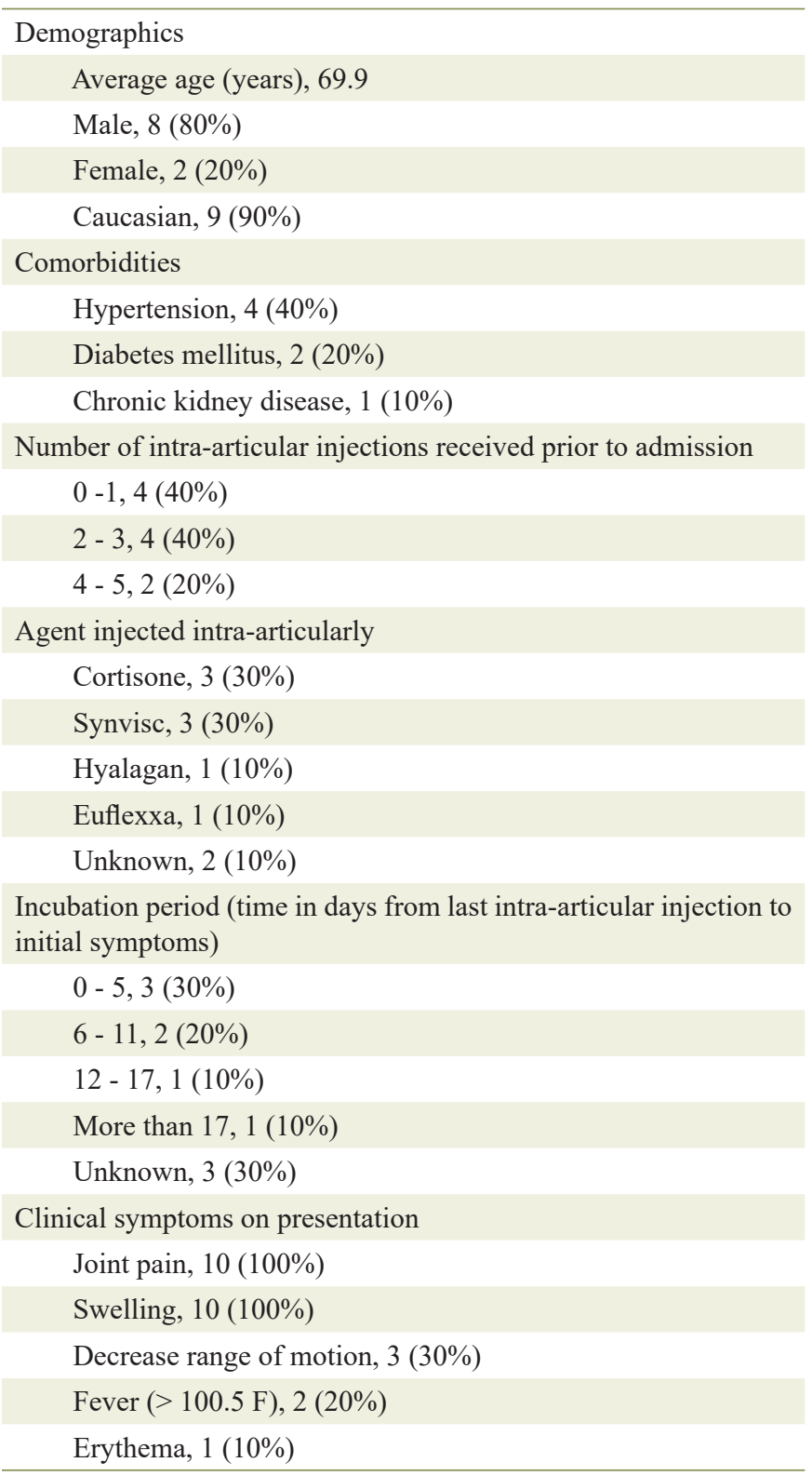

days. All patients underwent surgical intervention for septic arthritis. Three of ten patients needed more than one incision and drainage of the involved joint, with one patient needing three incision and drainages. Eight of ten patients were treated with antibiotics for 21 days. Five patients were treated with ceftriaxone, two with vancomycin and one with oral amoxicillinclavulinic therapy. Seven of ten patients received a PICC line for outpatient antibiotic therapy. Six patients required placement at rehabilitation facility.

One patient was re-admitted for bacteremia with Gemella morbilliform. This patient's first admission had a negative culture and antibiotics were stopped upon discharge. On second admission he was admitted with septic shock. He was started on 
Table 2. Characteristics of Synovial Fluid $(n=10)$

\begin{tabular}{|c|c|c|c|c|c|c|}
\hline $\begin{array}{l}\text { Patient } \\
\text { number }\end{array}$ & $\begin{array}{l}\text { ESR } \\
(\mathrm{mm} \text { in } \\
\text { first h) }\end{array}$ & $\begin{array}{l}\text { CRP refer- } \\
\text { ence value }(0 \\
-0.74 \mathrm{mg} / \mathrm{dL})\end{array}$ & $\begin{array}{l}\text { Appearance of } \\
\text { synovial fluid }\end{array}$ & $\begin{array}{l}\text { Fluid WBC count, } \\
\text { reference value } \\
(0-1,000 \text { cells/UL) }\end{array}$ & Crystals & Result of synovial fluid culture \\
\hline 1 & 111 & 26 & Turbid, yellow & 39,850 & $\begin{array}{l}\text { Calcium pyrophosphate } \\
\text { dihydrate }\end{array}$ & Streptococcus mitis/oralis \\
\hline 2 & 60 & 2.99 & Cloudy, yellow & 97,250 & $\begin{array}{l}\text { Calcium pyrophosphate } \\
\text { dihydrate }\end{array}$ & $\begin{array}{l}\text { Streptococcus mitis/ } \\
\text { oralis/Actinomyces odontolyticus }\end{array}$ \\
\hline 4 & 71 & 17.85 & Turbid, yellow & 11,933 & - & $\begin{array}{l}\text { Cx was negative on the first } \\
\text { admission, Gemella morbilliform } \\
\text { on the second admission }\end{array}$ \\
\hline 5 & 10 & 1.95 & Cloudy, yellow & 18,336 & - & No growth \\
\hline 6 & 51 & 5.9 & Turbid, yellow & - & Urate & Abiotrophia defectiva \\
\hline 9 & 76 & 14.6 & Cloudy, yellow & 98,901 & - & No growth \\
\hline 10 & 58 & 1.9 & Turbid, red & 15,380 & - & Streptoccus gordonii \\
\hline
\end{tabular}

ESR: erythrocyte sedimentation rate; CRP: C-reactive protein; WBC: white blood cell.

vancomycin and ceftriaxone. Because Gemella morbilliform is a potential cause for endocarditis, patient had a transesophageal echocardiography (TEE) which was non-conclusive in ruling out endocarditis. Patient was continued on intravenous antibiotics (vancomycin) for 4 weeks.

\section{Discussion}

In our case review, we describe 10 of the 41 cases of septic arthritis caused by intra-articular injection reported by Ross et al at an outpatient center in NJ [11]. Typically intra-articular joint injections have side effects reported in $2-10 \%$ of cases, with most common being injection site inflammation [8]. The incidence of septic arthritis caused by intra-articular injection incidence is about $13.3 \%$ of all septic arthritis [12].

Unlike most septic arthritis, which is primarily caused by Staph aureus and few gram negative organisms, all organisms identified at this facility were part of normal oral flora. In a case series review by $\mathrm{Xu}$ et al, the most common organism identified in septic arthritis after intra-articular injection was coagulase-negative staph, which is a common organism seen in skin flora [8]. Xu et al described tissue coring with epidermis and dermis into the joint as the likely pathogenesis behind this [8]. Another two case series of septic arthritis caused by intraarticular injections report that one patient had a staph aureus infection, and the second one had Neisseria mucosa [9]. In the case series review conducted by Ross et al, which included our ten patients, septic arthritis was deemed to be due to poor sterile techniques utilized while performing intra-articular injections [11]. According to Department of Health (DOH) report [11], the main reason outlined for this outbreak was poor compliance with standard infection control precautions during intra-articular injections including unhygienic preparation of the injection, lack of hand hygiene during administration, use of multidose vial of local anesthetic for multiple patients, intra-articular administration by general physician without special training, and high volume of patients [13].

Four out of 10 patients did not have growth on synovial fluid cultures, and one of these patients had received empirical antibiotics prior to joint tap. Inability to identify organisms in septic arthritis is fairly common, with literature suggesting its occurrence as nearly $58.8 \%$ [14-15]. This false negative result can be a challenge for physicians as it can affect appropriate therapy, leading to further clinical complications. Pseudoseptic arthritis is rare complication of intra-articular injections which should also be considered in appropriate clinical settings [16]. Pseudoseptic arthritis is an inflammatory arthritis which presents about $72 \mathrm{~h}$ after intra-articular injection. Symptoms are similar to septic arthritis; however joint cultures are sterile but inflammatory [16]. Mechanisms of injury are not fully understood. The diagnosis of pseudoseptic arthritis is usually made by exclusion of other causes.

An interesting observation of our ID physicians reviewing this outbreak is that interstate healthcare facilities often do not fall under jurisdiction of county or state DOH. Health departments can make recommendations but cannot compel private facilities to close. In this case, the outpatient facility reopened soon after their state inspection was completed.

\section{Conclusions}

Intra-articular injections to treat osteoarthritis typically are associated with minor side effects and a good alternative of pain control in opioid epidemic. But due to improper technique, 
poor procedure preparation including early setup of injections and inadequate training of personnel, this simple procedure can be associated with serious complications reflected in this case series. Internists especially primary care physicians should refer their patients needing these injections to appropriate providers such as orthopedic surgeons or rheumatologists.

\section{Acknowledgments}

We would thank Dr. Arthur Mark (orthopedics) for his early reporting of these cases as well as his management of multiple patients, the department of orthopedics at JSUMC for performing debridement surgeries on these patients and the Infection Prevention team at JSUMC for collecting data on these patients.

\section{Financial Disclosure}

This project was not supported by any grant or funding agencies.

\section{Conflict of Interest}

The authors have no conflict of interest to declare.

\section{Informed Consent}

Not applicable.

\section{Author Contributions}

SP, KP and MM contributed with patients' chart review, data collection and writing the initial draft for this manuscript; EL, $\mathrm{KC}$ and $\mathrm{MAH}$ contributed with reviewing and editing the final manuscript.

\section{References}

1. Mathews CJ, Coakley G. Septic arthritis: current diagnostic and therapeutic algorithm. Curr Opin Rheumatol. 2008;20(4):457-462.

2. Helito CP, Teixeira PR, Oliveira PR, Carvalho VC, Pecora JR, Camanho GL, Demange MK, et al. Septic arthritis of the knee: clinical and laboratory comparison of groups with different etiologies. Clinics (Sao Paulo). 2016;71(12):715-719.

3. Gupta MN, Sturrock RD, Field M. A prospective 2-year study of 75 patients with adult-onset septic arthritis.
Rheumatology (Oxford). 2001;40(1):24-30.

4. Sharp JT, Lidsky MD, Duffy J, Duncan MW. Infectious arthritis. Arch Intern Med. 1979;139(10):1125-1130.

5. Weston VC, Jones AC, Bradbury N, Fawthrop F, Doherty M. Clinical features and outcome of septic arthritis in a single UK Health District 1982-1991. Ann Rheum Dis. 1999;58(4):214-219.

6. Meijers KA, Dijkmans BA, Hermans J, van den Broek PJ, Cats A. Non-gonococcal infectious arthritis: a retrospective study. J Infect. 1987;14(1):13-20.

7. Kaandorp CJ, Dinant HJ, van de Laar MA, Moens HJ, Prins AP, Dijkmans BA. Incidence and sources of native and prosthetic joint infection: a community based prospective survey. Ann Rheum Dis. 1997;56(8):470-475.

8. Xu C, Peng H, Li R, Chai W, Li X, Fu J, Liu K, et al. Risk factors and clinical characteristics of deep knee infection in patients with intra-articular injections: A matched retrospective cohort analysis. Semin Arthritis Rheum. 2018;47(6):911-916.

9. Albert C, Brocq O, Gerard D, Roux C, Euller-Ziegler L. Septic knee arthritis after intra-articular hyaluronate injection. Two case reports. Joint Bone Spine. 2006;73(2):205207.

10. Bannuru RR, Natov NS, Dasi UR, Schmid CH, McAlindon TE. Therapeutic trajectory following intra-articular hyaluronic acid injection in knee osteoarthritis - metaanalysis. Osteoarthritis Cartilage. 2011;19(6):611-619.

11. Ross K, Mehr J, Carothers B, Greeley R, Benowitz I, McHugh L, Henry D, et al. Outbreak of Septic Arthritis Associated with Intra-Articular Injections at an Outpatient Practice - New Jersey, 2017. MMWR Morb Mortal Wkly Rep. 2017;66(29):777-779.

12. Garcia-Arias M, Balsa A, Mola EM. Septic arthritis. Best Pract Res Clin Rheumatol. 2011;25(3):407-213. United states Pharmacopeial convention. Chapter 797: pharmaceutical compounding-sterile preparations. In: United States Pharmacopeia 39 National Formulary 34. Rockville, MD. United States Pharmacopeial Convention; 2016:626-670

13. United States Pharmacopeial convention. Chapter 797: pharmaceutical compounding-sterile preparations. In: United States Pharmacopeia 39 National Formulary 34. Rockville, MD. United States Pharmacopeial Convention. 2016:626-670

14. Eberst-Ledoux J, Tournadre A, Mathieu S, Mrozek N, Soubrier M, Dubost JJ. Septic arthritis with negative bacteriological findings in adult native joints: a retrospective study of 74 cases. Joint Bone Spine. 2012;79(2):156-159.

15. Madruga Dias J, Costa MM, Pereira da Silva JA, Viana de Queiroz M. Septic arthritis: patients with or without isolated infectious agents have similar characteristics. Infection. 2014;42(2):385-391.

16. Idrissi Zineb, et al. Acute pseudo-septic arthritis following viscosuplementation of the knee. The Pan African Medical Journal. 2012;12(44):1-3. 